\title{
Nonlinear Mixed Effects Model Analysis of the Pharmacokinetics of Aripiprazole in Healthy Japanese Males
}

\author{
Toshiko Koue,${ }^{a, b}$ Masanori Kubo, ${ }^{a}$ Tomoo Funaki, ${ }^{a}$ Tsuyoshi Funuda, ${ }^{c}$ Junichi Azuma, ${ }^{c}$ \\ Mari TAKAAI, ${ }^{b}$ Yuichiro KAYANO, ${ }^{b}$ and Yukiya HASHIMOTO ${ }^{* b}$ \\ ${ }^{a}$ Department of Clinical Research and Development, Otsuka Pharmaceutical Co., Ltd.; Osaka 540-0021, Japan: \\ ${ }^{b}$ Graduate School of Pharmaceutical Sciences, University of Toyama; 2630 Sugitani, Toyama 930-0194, Japan: and \\ ${ }^{c}$ Department of Clinical Evaluation of Medicines and Therapeutics, Graduate School of Pharmaceutical Sciences, Osaka \\ University; Osaka 565-0871, Japan. Received June 5, 2007; accepted September 3, 2007
}

\begin{abstract}
The population pharmacokinetic parameters of aripiprazole in healthy Japanese males were estimated using a nonlinear mixed effects model (NONMEM) program. Pharmacokinetic data for population analysis were obtained from the single-dose ( 24 subjects), multiple-dose (15 subjects), and itraconazole-coadministration (27 subjects) trials. The time course of plasma aripiprazole concentration following oral administration was well described by a two-compartment model with first-order input. The mean values of the absorption lag time $(A L A G)$ and absorption rate constant $(K A)$ were estimated to be $0.805 \mathrm{~h}$ and $2.65 \mathrm{~h}^{-1}$, respectively. The mean volume of the central $\left(V_{1} / F\right)$ and peripheral $\left(V_{2} / F\right)$ compartment was 3.84 and $1.54 \mathrm{l} / \mathrm{kg}$, respectively, and the mean value of inter-compartment clearance $(Q / F)$ was $0.168 \mathrm{I} / \mathrm{h} / \mathrm{kg}$. Oral clearance $(C L / F)$ was estimated to be $0.0645 \mathrm{l} / \mathrm{h} / \mathrm{kg}$ in the group with $C Y P 2 D 6 * 1 / * 1, * 1 / * 2$ and $* 2 / * 2$. The decrease in $C L / F$ was estimated to be $0.01351 / \mathrm{h} / \mathrm{kg}$ in the group with $C Y P 2 D 6 * 1 / * 5, * 1 / * 10, * 2 / * 5, * 2 / * 10$, and $* 2 / * 41$, and $0.0293 \mathrm{l} / \mathrm{h} / \mathrm{kg}$ in the group with $C Y P 2 D 6 * 5 / * 10$, $* 10 / * 10$, and $* 41 / * 41$. The plasma concentration of aripiprazole was increased by coadministration of itraconazole, and the decrease in $C L / F$ was estimated to be $0.0181 \mathrm{l} / \mathrm{h} / \mathrm{kg}$.
\end{abstract}

Key words aripiprazole; two-compartment model; itraconazole; CYP2D6 genotype; nonlinear mixed-effect model; pharmacokinetic

Aripiprazole is a new antipsychotic developed in Japan, and is prescribed for schizophrenia as a dopamine-serotonin system stabilizer. ${ }^{1,2)}$ The action of aripiprazole is different from that of other typical and atypical antipsychotics; that is, aripiprazole is a potent partial agonist at dopamine D2 and serotonin $\left(5-\mathrm{HT}_{1 \mathrm{~A}}\right)$ receptors and antagonist at $5-\mathrm{HT}_{2 \mathrm{~A}}$ receptor. $^{3,4)}$ The results of in vitro studies indicated that aripiprazole is mainly metabolized by human cytochrome P450 isozymes CYP3A4 and CYP2D6. ${ }^{5}$ It has been reported that CYP3A4 and CYP2D6 are metabolic enzymes for numerous compounds, and also that there are many compounds that inhibit these enzymes. Although individual differences in hepatic levels of CYP3A4 enzyme protein have been reported to vary as much as 40 -fold, $\left.{ }^{6}\right)$ almost no gene mutations affecting CYP3A4 metabolic activity or ethnic differences have been reported. For CYP2D6, however, a number of polymorphisms and the existence of ethnic differences in the types and distribution of polymorphisms have been reported. ${ }^{67)}$ The purpose of the present study was to estimate the population pharmacokinetic parameters of aripiprazole in healthy Japanese males, and to evaluate the effects of CYP2D6 polymorphisms and CYP3A4 inhibition on the pharmacokinetics of aripiprazole, and also its unknown (residual) interindividual variability.

In the present study, we used the nonlinear mixed-effects model (NONMEM) method designed for the estimation of population pharmacokinetic parameters. ${ }^{8)}$ This method pools data from all individuals but explicitly models and handles the complicated error structure arising from proper accounting of interindividual $(\eta)$ and intraindividual $(\varepsilon)$ random effects. The first-order estimation method is the first estimation method available with NONMEM: it handles the error structure of data using first-order Taylor-series expansion in the random effect $\eta$, evaluated at the expected values (i.e. $\eta=0) .{ }^{9)}$ On the other hand, a newer analysis method designed for the estimation of population parameters, the firstorder conditional estimation (FOCE) method, is available with NONMEM software. ${ }^{9)}$ That is, FOCE uses first-order expansions for values of the $\eta$, but these values are the conditional (Bayesian) estimates of the $\eta$, rather than zero. The FOCE method needs much (20-40-fold) longer computational time than the first-order method, but generally gives precise parameter estimates, especially for the analysis of multiple-dose pharmacokinetic data with multiple compartment models. $^{9-12)}$ In the present study, therefore, we used the FOCE method to estimate the population pharmacokinetic parameters of aripiprazole, where a two-compartment model with first-order input was applied for data obtained from single- and multiple-dose clinical trials.

\section{MATERIALS AND METHODS}

Pharmacokinetic Data Plasma aripiprazole concentration data for population pharmacokinetic analysis were obtained in two previous studies. ${ }^{13,14)}$ Briefly, 68 healthy Japanese male subjects, aged 20-32 years old (mean: 23.1) and weighing $48.2-75.2 \mathrm{~kg}$ (mean: 60.7), participated in three clinical trials. Twenty-six subjects participated in the singledose (protocol 1) trial; 15 subjects participated in the multiple-dose (protocol 2) trial; and 27 subjects participated in the itraconazole-coadministration (protocol 3) trial. Genotyping data were also obtained in previous studies, where CYP2D $6 * 2, * 4, * 5, * 10, * 14, * 18, * 36$, and $* 41$ were detected by PCR-RFLP and long-PCR methods. ${ }^{13,14)}$ However, genotyping for CYP3A5 was not performed in the previous studies. $^{13,14)}$ Table 1 summarizes the genotypes of CYP2D6 in 68 Japanese subjects, who were divided into three groups according to the number of mutant (defective) alleles of 
Table 1. Genotypes of CYP2D6 in 68 Japanese Healthy Subjects

\begin{tabular}{|c|c|c|c|}
\hline & \multicolumn{3}{|c|}{ Protocol } \\
\hline & 1 & 2 & 3 \\
\hline \multicolumn{4}{|l|}{ Group 1} \\
\hline$* 1 / * 1$ & 3 & 2 & 4 \\
\hline$* 1 / * 2$ & 2 & 2 & - \\
\hline$* 2 / * 2$ & - & 1 & - \\
\hline \multicolumn{4}{|l|}{ Group 2} \\
\hline$* 1 / * 5$ & 1 & 1 & 4 \\
\hline$* 1 / * 10$ & 7 & 6 & 10 \\
\hline$* 2 / * 5$ & - & 1 & - \\
\hline$* 2 / * 10$ & 1 & 1 & 6 \\
\hline$* 2 / * 41$ & 1 & - & - \\
\hline \multicolumn{4}{|l|}{ Group 3} \\
\hline$* 5 / * 10$ & 5 & - & - \\
\hline$* 10 / * 10$ & 5 & 1 & 3 \\
\hline$* 41 / * 41$ & 1 & - & - \\
\hline
\end{tabular}

CYP2D6. ${ }^{15)}$ That is, Group 1 consisted of extensive metabolizers with $C Y P 2 D 6^{*} 1 / * 1, * 1 / * 2$, and $* 2 / * 2$; Group 2 consisted of subjects with $C Y P 2 D 6^{*} 1 / * 5, * 1 / * 10, * 2 / * 5, * 2 / * 10$, and $* 2 / * 41$; and Group 3 consisted of subjects with CYP2D6*5/*10,*10/*10, and *41/*41 (Table 1). ${ }^{15,16)}$ In addition, subjects heterozygous for CYP $2 D 6^{*} 2 / * 41$ and homozygous for $C Y P 2 D 6^{*} 41 / * 41$ were classified into Group 2 and Group 3, respectively, since it was reported that the $C Y P 2 D 6^{*} 41$ allele may be associated with the intermediate metabolizer phenotype. ${ }^{16,17)}$

In the single-dose (protocol 1) trial, each subject received a single dose of $6 \mathrm{mg}$ aripiprazole (ABILIFY ${ }^{\circledR}$ tablet) under a fasting condition. Blood samples were collected for the analysis of aripiprazole at 1, 2, 3, 4, 5, 6, 8, 12, 24, 48, 72, and $168 \mathrm{~h}$ after the dose. In the multiple-dose (protocol 2) trial, each subject received a once-daily dose of $3 \mathrm{mg}$ aripiprazole at $30 \mathrm{~min}$ after breakfast for 14 consecutive days. Blood samples were collected at 1, 2, 3, 4, 6, 8, and $12 \mathrm{~h}$ after the first dose on Day 1, at predose on Day 2-7, at 1, 2, 3, 4, 6,8 , and $12 \mathrm{~h}$ after the dose on Day 7 , and at predose on Day 8-14. After the last dose on Day 14, blood samples were collected at 1, 2, 3, 4, 6, 8, 12, 24, 48, 72, 96, 120, 168, 216, and $288 \mathrm{~h}$. In the protocol-3 trial, each subject first received a single dose of aripiprazole alone at $3 \mathrm{mg}$ once under a fasting condition. After a 5-week washout period, the subjects were administrated itraconazole at $100 \mathrm{mg}$ once daily for 21 consecutive days. Aripiprazole was administrated at the dose of $3 \mathrm{mg}$ on $7 \mathrm{~d}$ after the start of the administration of itraconazole. Blood samples were collected at 1, 2, 3, 4, 5, 6, 8, 12, $24,48,72,144,240$, and $366 \mathrm{~h}$ after the dose of aripiprazole. Plasma aripiprazole concentrations were measured using the LC-MS/MS method. ${ }^{13,14)}$

All subjects gave written consent to participate in the study, which was approved by the institutional review board of Osaka Pharmacology Clinical Research Hospital.

NONMEM Analysis of the Pharmacokinetics of Aripiprazole Population pharmacokinetic parameters were estimated using NONMEM software, ${ }^{9)}$ for which we used the FOCE in the present study. The two-compartment model with first-order absorption was parameterized in terms of absorption lag time $(A L A G)$, absorption rate constant $(K A)$, the apparent volume of central compartment $\left(V_{1} / F\right)$, oral clear- ance $(C L / F)$, the apparent volume of peripheral compartment $\left(V_{2} / F\right)$, and the apparent inter-compartment clearance $(Q / F)$ with NONMEM-PREDPP library subroutines, ADVAN4 and TRANS4. ${ }^{9)}$ The absorption lag time in the $i$ th individual $\left(A L A G_{i}\right)$ was modeled using the following equation:

$$
A L A G_{i}=\theta_{1} \cdot \exp \left(\eta_{A L A G_{i}}\right)
$$

where $\theta_{1}$ is the predicted population mean of the absorption lag time, and $\eta_{A L A G}$ is a random variable distributed with a mean of zero and variance of $\omega_{A L A G}^{2}$. The absorption rate constant in the $i$ th individual $\left(K A_{i}\right)$ was modeled using the following equation:

$$
K A_{i}=\theta_{2} \cdot \exp \left(\eta_{K A_{i}}\right)
$$

where $\theta_{2}$ is the predicted population mean of the absorption rate constant, and $\eta_{K A_{i}}$ is a random variable distributed with a mean of zero and variance of $\omega_{K A}^{2}$. The apparent volume of central compartment in the $i$ th individual $\left(V_{1} / F_{i}\right)$ was modeled using the following equation:

$$
V_{1} / F_{i}=\theta_{3} \cdot W T_{i} \cdot \exp \left(\eta_{V_{1} / F_{i}}\right)
$$

where $\theta_{3}$ is the predicted population mean of the apparent volume of the central compartment, $W T_{i}$ is the individual body weight, and $\eta_{V_{1} / F_{i}}$ is a random variable distributed with a mean of zero and variance of $\omega_{V_{1} / F}^{2}$. Oral clearance in the $i$ th individual $\left(C L / F_{i}\right)$ was modeled using the following equation:

$$
C L / F_{i}=\left(\theta_{4}-\theta_{5} \cdot \mathrm{G} 2-\theta_{6} \cdot \mathrm{G} 3-\theta_{7} \cdot \mathrm{ITZ}\right) \cdot W T_{i} \cdot \exp \left(\eta_{C L / F_{i}}\right)
$$

where $\theta_{4}$ is the predicted population mean of oral clearance, $\theta_{5}$ and $\theta_{6}$ are the clearance differences for Group 2 and Group 3, respectively, ${ }^{15)}$ and $\theta_{7}$ is the difference of the clearance when itraconazole was coadministrated. G2 is fixed to one for Group 2, and to zero for Group 1 and Group 3. G3 is fixed to one for Group 3, and to zero for Group 1 and Group 2. ITZ is fixed to one for the coadministration of itraconazole, and otherwise to zero. $\eta_{C L / F_{i}}$ is a random variable distributed with a mean of zero and variance of $\omega_{C L / F}^{2}$. The apparent volume of the peripheral compartment in the $i$ th individual $\left(V_{2} / F_{i}\right)$ was modeled using the following equation:

$$
V_{2} / F_{i}=\theta_{8} \cdot W T_{i} \cdot \exp \left(\eta_{V_{2} / F_{i}}\right)
$$

where $\theta_{8}$ is the predicted population mean of the apparent volume of the peripheral compartment, and $\eta_{V_{2} / F_{i}}$ is a random variable distributed with a mean of zero and variance of $\omega_{V / F}^{2}$. The apparent inter-compartment clearance in the $i$ th individual $\left(Q / F_{i}\right)$ was modeled using the following equation:

$$
Q / F_{i}=\theta_{9} \cdot W T_{i} \cdot \exp \left(\eta_{Q / F_{i}}\right)
$$

where $\theta_{9}$ is the predicted population mean of the apparent volume of inter-compartment clearance, and $\eta_{Q / F_{i}}$ is a random variable distributed with a mean of zero and variance of $\omega_{O / F}^{2}$. Finally, the $j$ th observed plasma concentration in the $i$ th subject $\left(C_{i j}\right)$ was assumed to be randomly and normally distributed from the predicted value $\left(C_{i j}^{*}\right)$ :

$$
C_{i j}=C_{i j}^{*} \cdot \exp \left(\varepsilon_{i j}\right)
$$

where $\varepsilon_{i j}$ is a random variable that describes intraindividual variability with a mean of zero and variance of $\sigma^{2}$. NONMEM provides estimates of the standard error (S.E.) for all parameters, and S.E. can be used to define 95\% confidence 


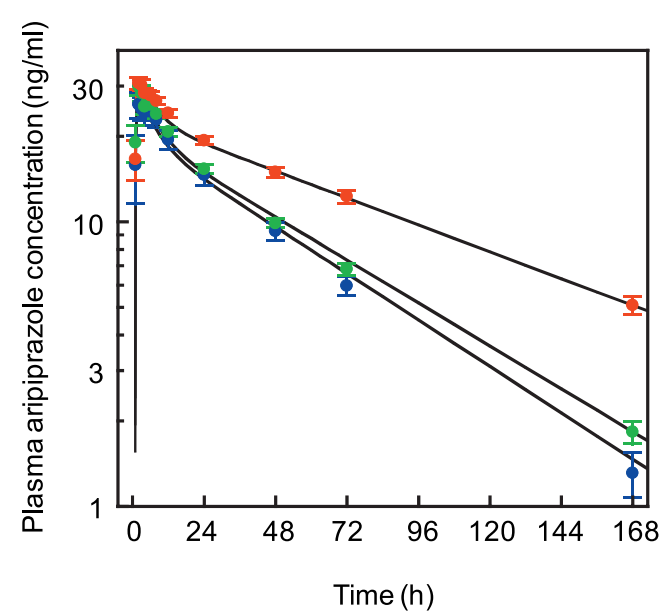

Fig. 1. Plasma Aripiprazole Concentration Following Oral Administration at a Dose of $6 \mathrm{mg}$

Blue circles: Group $1(n=5)$, green circles: Group $2(n=10)$, red circles: Group $3(n=11)$. The solid curve represents the predicted aripiprazole concentration in a typical subject having mean drug concentration in each group, where the pharmacokinetic parameters in the subject were obtained from population estimates according to Bayes' theorem using the NONMEM post-hoc option. Each symbol and bar represents the mean \pm S.E.

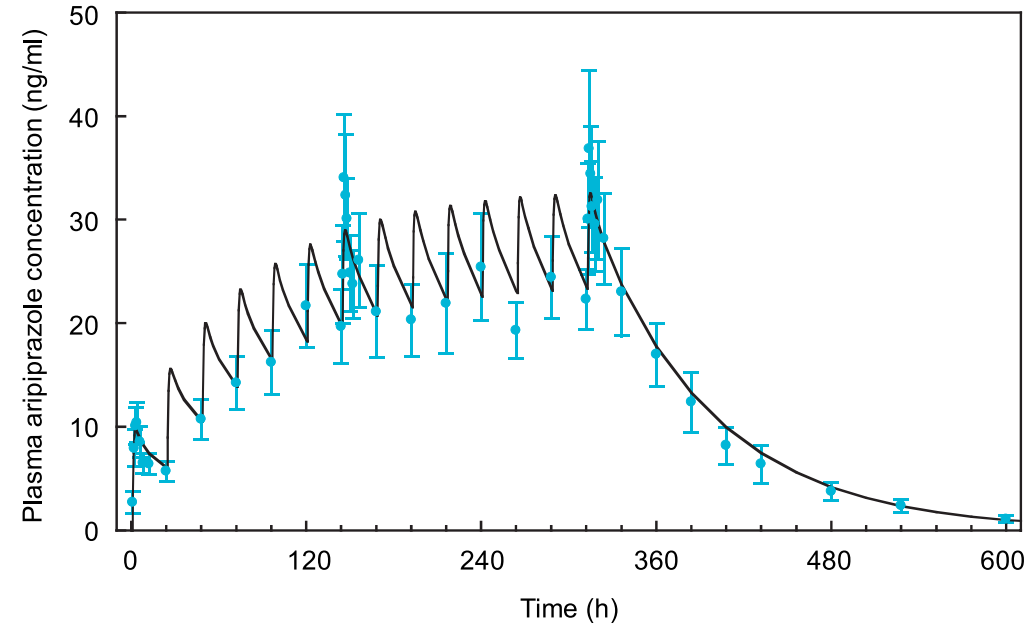

Fig. 2. Plasma Aripiprazole Concentration Following Repetitive Oral Administration to 15 Subjects at $3 \mathrm{mg}$ Once Daily for $14 \mathrm{~d}$

The solid curve represents the predicted aripiprazole concentration in a typical subject having mean drug concentration in 15 subjects, where the pharmacokinetic parameters in the subject were obtained from population estimates according to Bayes' theorem using the NONMEM post-hoc option. Each symbol and bar represents the mean \pm S.E. for 15 subjects. intervals (CI) for true parameter values: $95 \% \mathrm{CI}=($ estimated parameter value $) \pm 1.96 \cdot$ S.E. $^{9)}$

\section{RESULTS AND DISCUSSION}

Our purpose in the present study was to estimate the population pharmacokinetic parameters of aripiprazole, and also to evaluate the effects of CYP2D6 polymorphisms and itraconazole-coadministration on the pharmacokinetics of the drug. Pharmacokinetic data for population analysis were obtained in three previous clinical trials. ${ }^{13,14)}$ The subjects were 26 healthy Japanese males in the single-dose trial, 15 males in the multiple-dose trial, and 27 males in the itraconazolecoadministration trial. Population pharmacokinetic analysis was performed using NONMEM software, because NONMEM analysis enables us to simultaneously evaluate mean pharmacokinetic parameters, the covariates affecting the pharmacokinetics of a drug, and also unknown interindividual pharmacokinetic variability.

Figure 1 shows plasma aripiprazole concentrations following oral administration at a dose of $6 \mathrm{mg}$ in the single-dose trial. Concentration-time curves in the three genotype groups were obtained by pharmacokinetic analysis using a two-compartment model with first-order absorption. The absorption lag time of aripiprazole was very short, and then plasma concentrations rose sharply, indicating extremely rapid absorption of aripiprazole. Plasma aripiprazole concentrations in Group 2 were only slightly higher than those in Group 1, whereas plasma concentrations in Group 3 were significantly higher than those in Group 1. The mean terminal half-lives of the drug in Group 1, Group 2, and Group 3 were calculated to be $44.2,47.7,76.3 \mathrm{~h}$, respectively (Fig. 1). Figure 2 shows the mean plasma aripiprazole concentration-time curve following repetitive oral administration at a dose of $3 \mathrm{mg}$. The concentrations of aripiprazole rose sharply despite drug administration after breakfast, indicating that the effect of food on the gastrointestinal absorption of aripiprazole was

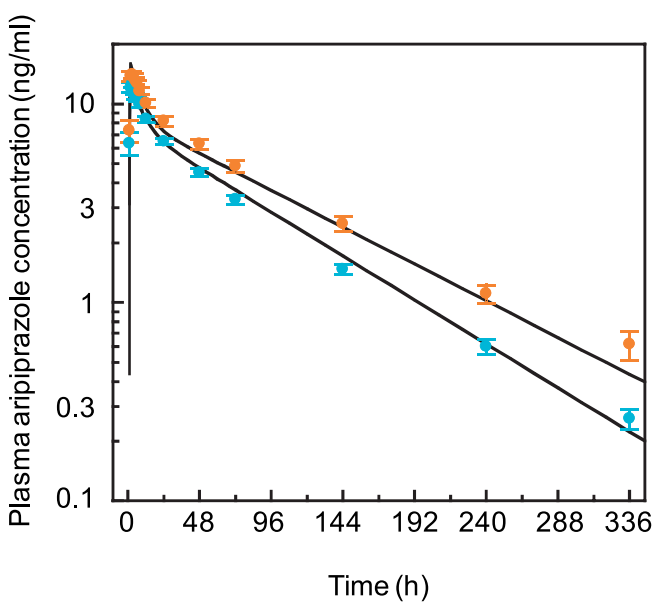

Fig. 3. Effect of Coadministrated Itraconazole on Plasma Aripiprazole Concentration Following Oral Administration to 27 Subjects at a Dose of $3 \mathrm{mg}$

Light blue circles: aripiprazole alone, orange circles: aripiprazole with itraconazole. The solid curve represents the predicted aripiprazole concentration in a typical subject having mean drug concentration in 27 subjects, where the pharmacokinetic parameters in the subject were obtained from population estimates according to Bayes' theorem using the NONMEM post-hoc option. Each symbol and bar represents the mean \pm S.E.

not significant. The time course of plasma aripiprazole concentration reached a quasi-steady-state at $10-14 \mathrm{~d}$ following the repetitive once-daily administration of the drug. Figure 3 shows the effect of coadministrated itraconazole, a potent inhibitor of CYP3A4, on plasma aripiprazole concentrations. Plasma aripiprazole concentrations in the itraconazole-coadministration period were significantly higher than those in the period without itraconazole. The terminal half-lives of aripiprazole without and with coadministration of itraconazole were calculated to be 65.2 and $77.9 \mathrm{~h}$, respectively. Although CYP2D6 polymorphisms and coadministration of itraconazole induced a significant change of plasma aripiprazole concentrations, the predicted plasma concentrations of aripiprazole were well fitted to the observed plasma concentrations 
Table 2. Population Pharmacokinetic Parameters of Aripiprazole in Healthy Japanese Subjects

\begin{tabular}{llc}
\hline \hline Parameters & Estimates & $95 \% \mathrm{CI}$ \\
\hline$\theta_{1}(\mathrm{~h})$ & 0.805 & $0.738-0.872$ \\
$\theta_{2}\left(\mathrm{~h}^{-1}\right)$ & 2.65 & $1.92-3.38$ \\
$\theta_{3}(1 / \mathrm{kg})$ & 3.84 & $3.32-4.36$ \\
$\theta_{4}(1 / \mathrm{h} / \mathrm{kg})$ & 0.0645 & $0.0494-0.0796$ \\
$\theta_{5}(1 / \mathrm{h} / \mathrm{kg})$ & 0.0135 & $-0.0067-0.0337$ \\
$\theta_{6}(1 / \mathrm{h} / \mathrm{kg})$ & 0.0293 & $0.0070-0.0516$ \\
$\theta_{7}(1 / \mathrm{h} / \mathrm{kg})$ & 0.0181 & $0.0010-0.0352$ \\
$\theta_{8}(1 / \mathrm{kg})$ & 1.54 & $1.32-1.76$ \\
$\theta_{9}(1 / \mathrm{h} / \mathrm{kg})$ & 0.168 & $0.125-0.211$ \\
$\omega_{A L A G}$ & 0.110 & $0.036-0.151$ \\
$\omega_{K A}$ & 0.910 & $0.711-1.07$ \\
$\omega_{V_{1} / F}$ & 0.381 & $0.245-0.479$ \\
$\omega_{C L / F}$ & 0.397 & $0.295-0.479$ \\
$\omega_{V_{2} / F}$ & 0.365 & $0.185-0.481$ \\
$\omega_{Q / F}$ & 0.262 & $0-0.494$ \\
$\sigma$ & 0.166 & $0.124-0.200$ \\
\hline
\end{tabular}

of the drug in all three clinical trails (Figs. 1-3). These findings indicated that the pharmacokinetics of aripiprazole can be well described by a two-compartment model with firstorder absorption. In other words, no significant saturation and/or induction were observed in the intestinal absorption, tissue distribution, and/or hepatic metabolism of the drug. The pharmacokinetics of aripiprazole may be linear at the dose range tested $(3-6 \mathrm{mg} / \mathrm{d})$ and constant over a long period of time (at least $600 \mathrm{~h}$ ).

The population pharmacokinetic parameters of aripiprazole and their 95\% CI by NONMEM analysis are listed in Table 2. The mean value of $A L A G$ and $K A$ was estimated to be $0.805 \mathrm{~h}$ and $2.65 \mathrm{~h}^{-1}$, respectively. The mean value of $V_{1} / F$ and $V_{2} / F$ was 3.84 and $1.541 / \mathrm{kg}$, respectively, and the mean value of $Q / F$ was $0.168 \mathrm{l} / \mathrm{h} / \mathrm{kg}$. The mean value of $C L / F$ was estimated to be $0.0645 \mathrm{l} / \mathrm{h} / \mathrm{kg}$ in Group 1 . The decrease in $C L / F$ was estimated to be $0.0135 \mathrm{l} / \mathrm{h} / \mathrm{kg}$ in Group 2, and $0.02931 / \mathrm{h} / \mathrm{kg}$ in Group 3. The decrease in $C L / F$ was estimated to be $0.0181 \mathrm{l} / \mathrm{h} / \mathrm{kg}$ in the itraconazole coadministration period. On the other hand, large interindividual variability was observed not only in the absorption rate of aripiprazole, but also in the distribution and elimination of the drug (Table 2). That is, although the interindividual variability of $A L A G$ was small $\left(\omega_{A L A G}=0.110\right)$, that of $K A$ was very large $\left(\omega_{K A}=0.910\right)$. The $\omega_{V_{1} / F}, \omega_{V_{2} / F}$, and $\omega_{Q / F}$ values were estimated to be $0.381,0.365$, and 0.262 , respectively. The interindividual variability of $C L / F\left(\omega_{C L / F}\right)$ was also considerably large, and was estimated to be 0.397 (Table 2). Therefore, plasma aripiprazole concentration in individual subjects was often different from that predicted by the mean population pharmacokinetic parameters $\left(\theta_{1}-\theta_{9}\right)$ listed in Table 2 . Figure 4 shows the relationship between the observed and predicted concentrations of aripiprazole in 68 subjects. There was large interindividual variability in the observed concentration of aripiprazole (Fig. 4).

In general, the inter- and intra-individual variability of $K A$ is large. ${ }^{12)}$ In the case of aripiprazole, large interindividual variability was observed not only for $K A$, but also for $C L / F$, $V_{1} / F$, and $V_{2} / F$ (Table 2). Since absolute bioavailability $(F)$ of aripiprazole was reported to be $87 \%$, its absorption from the gastrointestinal tract should be almost complete. ${ }^{5)}$ In addition, the high value of $F$ of aripiprazole suggests that the

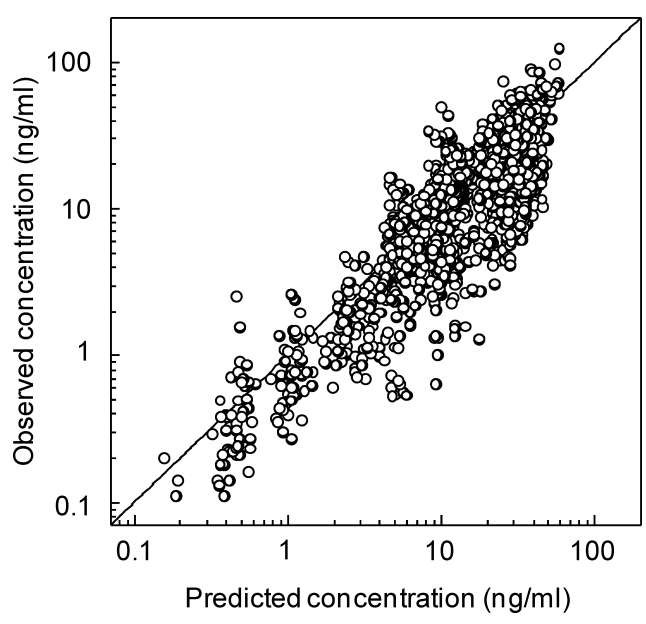

Fig. 4. The Relationship between the Observed and Predicted Concentration of Aripiprazole in 68 Subjects

first-pass extraction of the drug in the liver and intestine is not significant, and that the large interindividual variability in $C L / F, V_{1} / F$, and $V_{2} / F$ is not due to intestinal absorption and/or presystemic clearance. ${ }^{5}$ ) On the other hand, systemic clearance $(C L)$ of drugs primarily metabolized in the liver is influenced not only by the hepatic blood flow rate and hepatic intrinsic clearance $\left(C L_{\text {int }}\right)$, but also by the unbound fraction $\left(f_{\mathrm{u}}\right)$ in the blood (plasma). ${ }^{18-20)}$ The volume of drug distribution $(V)$ is also largely influenced by the interindividual variability of $f_{\mathrm{u}^{\cdot}}{ }^{21}$ ) It was reported that protein binding of aripiprazole was more than $99 \%$, and that the $f_{\mathrm{u}}$ value of the drug was very small. ${ }^{5)}$ The finding suggests that a slight change of protein binding of aripiprazole can lead to a large change of $f_{\mathrm{u}}$. Therefore, we thought that the large interindividual variability in $C L / F, V_{1} / F$, and $V_{2} / F$ may be, at least partly, due to high plasma protein binding of aripiprazole.

In conclusion, we analyzed the pharmacokinetics of aripiprazole using NONMEM software. Orally administrated aripiprazole was rapidly absorbed from the gastrointestinal tract, and the pharmacokinetics of aripiprazole was significantly altered by the polymorphism of CYP2D6 and by the coadministration of CYP3A4 inhibitor(s). However, the time course of plasma aripiprazole concentration following single and multiple oral administration was well described by a two-compartment model with first-order absorption, which suggested that the pharmacokinetics of the drug is linear and constant at the dose and time range tested. In addition, the pharmacokinetic parameters in individual patients can be obtained from the population pharmacokinetic parameters according to Bayes' theorem. The population pharmacokinetic parameters of aripiprazole estimated in the present study may be useful for studying the concentration-effect relationship of the drug.

\section{REFERENCES AND NOTES}

1) Oshiro Y., Sato S., Kurahashi N., Tanaka T., Kikuchi T., Tottori K., Uwahodo Y., Nishi T., J. Med. Chem., 41, 658-667 (1998).

2) El-Sayeh H. G., Morganti C., Adams C. E., Br. J. Psychiatry, 189, $102-108$ (2006)

3) Burris K. D., Molski T. F., Xu C., Ryan E., Tottori K., Kikuchi T., Yocca F. D., Molinoff P. B., J. Pharmacol. Exp. Ther, 302, 381-389 (2002). 
4) Jordan S., Koprivica V., Chen R., Tottori K., Kikuchi T., Altar C. A., Eur. J. Pharmacol., 441, 137-140 (2002).

5) Package insert of Abilify ${ }^{\circledR}$ (Aripiprazole, U.S.), April 2004 Revision, Otsuka America Pharmaceutical, Inc., Rockville, MD 20850, U.S.A. and Bristol-Myers Squibb Co., Princeton, NJ 08543, U.S.A.

6) Ozawa S., Soyama A., Saeki M., Fukushima-Uesaka H., Itoda M., Koyano S., Sai K., Ohno Y., Saito Y., Sawada J., Drug Metab. Pharmacokinet., 19, 83-95 (2004).

7) Nishida Y., Fukuda T., Yamamoto I., Azuma J., Pharmacogenetics, 10, $567-570(2000)$.

8) Sheiner L. B., Ludden T. M., Annu. Rev. Pharmacol. Toxicol., 32, 185-209 (1992)

9) Beal S. L., Boeckmann A. J., Sheiner L. B., "NONMEM Users Guides: NONMEM Project Group," University of California, San Francisco, 1992.

10) Hashimoto Y., Ozaki J., Koue T., Odani A., Yasuhara M., Hori R., Pharm. Res., 11, 545-548 (1994).

11) Hashimoto Y., Koue T., Otsuki Y., Yasuhara M., Hori R., Inui K., $J$. Pharmacokinet. Biopharm., 23, 205-216 (1995).
12) Honda M., Itoh H., Suzuki T., Hashimoto Y., Biol. Pharm. Bull., 29, 2460-2464 (2006).

13) Kubo M., Koue T., Inaba A., Takeda H., Maune H., Fukuda T., Azuma J., Drug Metab. Pharmacokinet., 20, 55-64 (2005).

14) Kubo M., Koue T., Maune H., Fukuda T., Azuma J., Drug Metab. Pharmacokinet., in press.

15) Taguchi M., Nozawa T., Mizumaki K., Inoue H., Tahara K., Takesono C., Hashimoto Y., Biol. Pharm. Bull., 27, 1642-1648 (2004).

16) Zanger U. M., Fischer J., Raimundo S., Stuven T., Evert B. O., Schwab M., Eichelbaum M., Pharmacogenetics, 11, 573-585 (2001).

17) Toscano C., Klein K., Blievernicht J., Schaeffeler E., Saussele T., Raimundo S., Eichelbaum M., Schwab M., Zanger U. M., Pharmacogenet. Genomics, 16, 755-766 (2006).

18) Rowland M., Benet L. Z., Graham G. G., J. Pharmacokinet. Biopharm., 1, 123-136 (1973).

19) Wilkinson G. R., Shand D. G., Clin. Pharmacol. Ther., 18, 377-390 (1975).

20) Wilkinson G. R., Pharmacol. Rev., 39, 1-47 (1987).

21) Øie S., Tozer T. N., J. Pharm. Sci., 68, 1203-1205 (1979). 